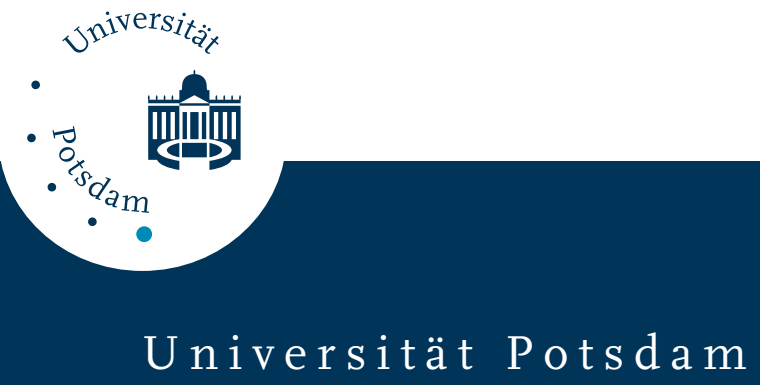

Sebastian Reich

\title{
On an existence and uniqueness theory for nonlinear differential-algebraic equations
}

first published in:

Circuits, Systems, and Signal Processing 10 (1991), 3, S. 343-359

Postprint published at the Institutional Repository of the Potsdam University:

In: Postprints der Universität Potsdam

Mathematisch-Naturwissenschaftliche Reihe ; 158

http://opus.kobv.de/ubp/volltexte/2010/4670/

http://nbn-resolving.de/urn:nbn:de:kobv:517-opus-46706

Postprints der Universität Potsdam

Mathematisch-Naturwissenschaftliche Reihe ; 158 


\title{
ON AN EXISTENCE AND UNIQUENESS THEORY FOR NONLINEAR DiffERENTIAL- Algeibraic Equations*
}

\author{
Sebastian Reich ${ }^{1}$
}

\begin{abstract}
An existence and uriqueness theory is developed for general nonlinear and nonautonomous differential-cilgebraic equations (DAEs) by exploiting their underlying differential-geometric structure. A DAE is called regular if there is a uniquz nonautonomous vector field such that the solutions of the DAE and the solutions cf the vector field are in one-to-one correspondence. Sufficient conditions for regularity of a DAE are derived in terms of constrained manifolds. Based on this differentia geometric characterization, existence and uniqueness results are stated for regular DAEs. Furthermore, our not ons are compared with techniques frequently used in the literature such as index and solvability. The resılts are illustrated in detail by means of a simple circuit example.
\end{abstract}

\section{Introduction}

Differential-algebraic equations (DAEs) c.re frequently identified as implicit equations

$$
F\left(t, x, x^{\prime}\right)=0
$$

for which $x^{\prime}$ cannot be expressed explicitly as a function of $t$ and $x$. Such DAEs arise in many areas of science and engineering. 'n particular, constrained mechanical systems and electronic circuits may be modeled using equations of type (1). In secent years the literature on the numerical solution of (1) has been growing rapidly [4], [10], [12], [14]. However, up to now, existence theories are available only for a few selected classes of DAEs which are characterized by restrictive condition:s on the form of the mapping $F$ in (1) (see, e.g., [16], [18], and [19] for autonomous DAEs and [4] for nonautonomous linear DAEs).

In [19] Rheinboldt introduced a differential-geometric approach for

* Received May 10, 1990; revised August 10, 199').

${ }^{1}$ Karl-Weierstrasse-Institut fïr Mathematik, Mohrenstrasse 39, D/O-1086 Berlin, Germany. 
analysis of the solution properties of a class of autonomous DAEs. This differential-geometric approach is based on the observation that, as long as we expect the solutions of a DAE to be some smooth path in the space of variables, the solutions define a flow [1] on a suitable submanifold of that space. Conversely, it is well known that flows are strongly related to vector fields on manifolds [1]. Thus we regard a DAE as an implicit description of a vector field, and have introduced in [17] and [18] the notion of a regular DAE as a DAE to which a vector field uniquely corresponds. The basic idea of this approach is the following one: Once the regularity of a DAE has been established, existence and uniqueness results can be obtained by employing the well-known existence theory of vector fields.

In this paper we wish to elaborate on the concept of regularity for general nonautonomous DAEs of type (1). After surveying the necessary background material, we derive in Section 3 sufficient conditions for the regularity of a DAE in terms of constrained manifolds. From this and the standard theory of vector fields, we formulate existence and uniqueness results for regular DAEs as a main result of this paper. Because, in the literature, DAEs are categorized by many other concepts such as index [4], [9], [14], [16] and solvability [4], [5], we provide a comparison between these various techniques in Section 5. Additionally we give a detailed discussion of a simple circuit which appears to illustrate our concepts.

For that reason let us consider throughout this paper the RC circuit of Figure 1. We assume that the voltage-current relation $R$ of the nonlinear resistor is given by

$$
R=\left\{\left(t, u_{R}, i_{R}\right) \in \mathbb{R} \times \mathbb{R}^{2}: 0=g\left(t, u_{R}, i_{R}\right)\right\}
$$

with $g: \mathbb{R} \times \mathbb{R}^{2} \rightarrow \mathbb{R}$ of class $C^{1}$ and $\operatorname{rank}\left[D_{2} g D_{3} g\right]\left(t, u_{R}, i_{R}\right)=1$ for all $\left(t, u_{R}, i_{R}\right) \in \mathbb{R} \times \mathbb{R}^{2}$ where $D_{2} g$ and $D_{3} g$ denote the partial derivatives of $g$ with respect to the second and third argument respectively. This implies that the set $R$ is a differentiable manifold and that the time behavior of the RC

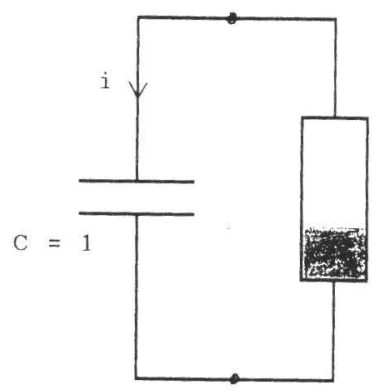

Figure 1. A simple RC circuit. 
circuit can be modeled by the DAE

$$
\begin{aligned}
& q^{\prime}=i, \\
& 0=g(t, q,-i) .
\end{aligned}
$$

\section{Differential-geometric background}

In this section we collect some basic material needed throughout the remainder of this presentation. Especially, we generalize the notions of vector fields and flows, as used in the standard literature, to the nonautonomous case and incorporate the fact that we consider in this paper only manifolds which are embedded in $\mathbb{R}^{n}$. For further details we refer the reader to standard texts on differential geometry such as [1] and [13].

We begin with some standard terminology. If $M$ is a differentiable manifold, then $T M$ denotes the tangent tundle of $M$ and $T_{x} M$ denotes the tangent space of $M$ at $x: M$. Throughout this paper we consider only manifolds which are embedded in $\mathbb{R}^{n}$; that is, submanifolds of $\mathbb{R}^{n}$. In accordance with this, we can always assure that the tangent space $T_{x} M$ can be identified with a $d$-dimensional linear subspace of $\mathbb{R}^{n}$ where $d=\operatorname{dim}(M)$.

Now let $M$ be a differentiable submanifold of $\mathbb{R} \times \mathbb{R}^{n}$, let $\mathrm{pr}_{1}: \mathbb{R} \times \mathbb{R}^{n} \rightarrow \mathbb{R}$ be the projection onto the first component in $\mathbb{R} \times \mathbb{R}^{n}$, let $J$ be an open set of $\mathbb{R}$ with $\operatorname{pr}_{1}(M)=J$, and let $p$ be the restriction of $\operatorname{pr}_{1}$ to $M$. Then the triple $(M, p, J)$ is a subbundle of $\mathbb{R} \times \mathbb{R}^{n}$ if $\operatorname{pr}_{1}\left(T_{(t, x)} M\right)=\mathbb{R}$ for all $(t, x) \in M$. For any subbundle $(M, p, J)$ the manifolds $M_{t} \subset \mathbb{R}^{n}$, defined by

$$
\{t\} \times M_{t}=\left(M \cap\{t\} \times \mathbb{R}^{n}\right) \quad(t \in J),
$$

are called the fibers of $M$ at $t$.

Let $f: J \times U \rightarrow \mathbb{R}^{m}$ be a given mapping of class $C^{r}, r>0$, on the open set $J \times U \subset \mathbb{R} \times \mathbb{R}^{n}$. Then ve denote by $D_{1} f(t, x)$ (resp. $\left.D_{2} f(t, x)\right)$ the partial derivatives of $f$ with respect to the first, (resp. second) argument. Now most classical examples of dif'erentiable subbundles of $\mathbb{R} \times \mathbb{R}^{n}$ can be identified with the zeros of differentiable mappings $f: J \times U \rightarrow \mathbb{R}^{m}$. Specifically:

Let $f: J \times U \rightarrow \mathbb{R}^{m}, d=n+1-m>0$, be a given mapping of class $\boldsymbol{c}^{r}$, $r>0$, on the open set $J \times U \subset \mathbb{R} \times \mathbb{R}^{n}$ such that $0 \in f(J \times U)$ and, for all $(t, x) \in J \times U, \operatorname{im}\left[D_{2} f(t, x)\right]=\mathbb{R}^{m}$. Then the triple $(M, p, J)$ with

$$
M=\{(t, x) \in J \times U: f(t, x)=0\}
$$

is a $C^{r}$-subbundle of $\mathbb{R} x \mathbb{R}^{n}$. The fibers of the bundle are given by

$$
M_{t}=\{x \in U: f(t, x)=0\} \quad(t \in J),
$$

and the tangent space of $M$ at $(t, x)$ is given by

$$
T_{(t, x)} M=\left\{\left(t^{\prime}, x^{\prime}\right) \in \mathbb{R} \times \mathbb{R}^{n}: D_{1} f(t, x) t^{\prime}+D_{2} f(t, x) x^{\prime}=0\right\} .
$$


The following definition is motivated by the fact that, for nonautonomous vector fields, every element $\left(t^{\prime}, x^{\prime}\right)$ of the tangent space $T_{(t, x)} M$ has to satisfy $t^{\prime}=1$. Thus, for any subbundle $(M, p, J)$ of $\mathbb{R} \times \mathbb{R}^{n}$, we define the sets $S_{(t, x)} M$ and $S M$ by

$$
S_{(t, x)} M=\left\{x^{\prime} \in \mathbb{R}^{n}:\left(1, x^{\prime}\right) \in T_{(t, x)} M\right\}
$$

and

$$
\mathrm{SM}=\bigcup_{(t, x) \in M}\{(t, x)\} \times S_{(t, x)} M
$$

which we call the restricted tangent space of $M$ at $(t, x)$ and the restricted . tangent bundle of $M$, respectively.

Example 1. For the circuit example of Figure 1 the voltage-current relation $R$ of the nonlinear resistor is a subbundle of $\mathbb{R} \times \mathbb{R}^{2}$. For a fixed $t \in \mathbb{R}$ the fiber $R_{t}$ is given by the solutions $\left(u_{R}, i_{R}\right)$ of

$$
g\left(t, u_{R}, i_{R}\right)=0 .
$$

Furthermore, the restricted tangent space $S_{\left(t, u_{R}, i_{R}\right)} R$ is characterized by the solutions $\left(u_{R}^{\prime}, i_{R}^{\prime}\right) \in \mathbb{R}^{2}$ of

$$
D_{1} g\left(t, u_{R}, i_{R}\right)+D_{2} g\left(t, u_{R}, i_{R}\right) i_{R}^{\prime}+D_{3} g\left(t, u_{R}, i_{R}\right) i_{R}^{\prime}=0
$$

for all $(t, u, i) \in R$.

Let $(M, p, J)$ be a $C^{r}$-subbundle, $r>0$, of $\mathbb{R} \times \mathbb{R}^{n}$. Then a mapping $v: M \rightarrow \mathbb{R}^{n}$ of class $C^{k}, k \geq 0$, is called a (nonautonomous) vector field of class $C^{k}$ on $M$ if $v(t, x) \in S_{(t, x)} M$ for all $(t, x) \in M$. By a solution of a vector field $v: M \rightarrow \mathbb{R}^{n}$ we mean a differentiable mapping $c: I \rightarrow \mathbb{R}^{n}$ on the open interval $I \subset J$ such that $c(t) \in M_{t}$ and

$$
D c(t)=v(t, c(t))
$$

for all $t \in I$.

Remark 1. In several papers (see, e.g., [1]) the subbundle $(M, p, J)$ is assumed . to be trivial; that is, $M=J \times Q$ where $Q$ is a submanifold of $\mathbb{R}^{n}$. However, subsequent discussion will reveal that we indeed need the more general definition of a nonautonomous vector field on a nontrivial subbundle $(M, p, J)$. Furthermore, the manifold $M$ cannot be viewed, in general, as a linear space by globally coordinatizing it; that is, the manifold $M$ cannot be described in general by just one chart.

Remark 2. With a given vector field $v: M \rightarrow \mathbb{R}^{n}$ we can associate the nonautonomous ODE

$$
x^{\prime}=v(t, x) \quad((t, x) \in M)
$$


on the manifold $M$. Thus the notions of a vector field and an ODE on a manifold are synonymous in case the manifold $M$ has been embedded in $\mathbb{R}^{\prime \prime}$.

Let $(M, p, J)$ be a $C^{r}$-subbundle, $r>0$, of $\mathbb{R} \times \mathbb{R}^{n}$ and let $D$ be an open set in $J \times M$. Then a mapping $H: D \rightarrow \mathbb{R}^{n}$ of c ass $C^{k}, k>0$, is called a flow [1] of class $C^{k}$ on $D$ if

(i) $\{0\} \times M \subset D$;

(ii) for all $\left(t_{0}, x_{0}\right) \in M, H\left(0 ; t_{0}, x_{0}\right)=x_{0}$;

(iii) for all $\left(t ; t_{0}, x_{0}\right) \in D, \quad\left(s ; t, H^{\prime} t ; t_{0}, x_{0}\right) \in D$ if and only if $\left(s+t ; t_{0}, x_{0}\right) \in D$; in this case

$$
H\left(s+t ; t_{0}, x_{0}\right)=H\left(s ; t, H\left(t ; t_{0}, x_{0}\right)\right) \text {. }
$$

- Theorem 1 [1]. Let $(M, p, J)$ be a subbundle of $\mathbb{R} \times \mathbb{R}^{n}$ and let $v: M \rightarrow \mathbb{R}^{n}$ be a vector field of class $C^{k}, k>0$. Then there is a unique open set $D$ in $J \times M$ and a unique flow $H: D \rightarrow \mathbb{R}^{n}$ of class $C^{k}$ on $D$ such that a differentiable mapping $c: I \rightarrow \mathbb{R}^{n}$ with $c\left(t_{0}\right)=x_{0}$ is a solution of the vector field $v$ if and only if

$$
c(t)=H\left(t ; t_{0}, x_{0}\right)
$$

for all $t \in I$.

Remark 3. Obviously the existence and inniqueness of the flow $H$ implies all the well-known results on the existence, uniqueness, unique extension, and dependence on initial values of solutions.

\section{Regular DAEs}

From now on we study DAEs of type

$$
F\left(t, x, x^{\prime},:=0,\right.
$$

where $F: J \times U \times \mathbb{R}^{n} \rightarrow \mathbb{R}^{n}$ is a mapping of class $C^{r}, r>0$, on the open set $J \times U \times \mathbb{R}^{n} \subset \mathbb{R} \times \mathbb{R}^{n} \times \mathbb{R}^{n}$. By a solution of a $D A E$ (3) we mean a mapping $c: I \rightarrow \mathbb{R}^{n}$ of class $C^{1}$ on the open interval $I \subset J$ such that, for all $t \in I$,

$$
F(t, c(t), D c(t))=0 .
$$

As suggested in the Introduction, we consider DAEs as an implicit description of vector fields on manifolds. In line with this we have propesed in [18] and [19] the notion of regularity for a special class of autonomous DAEs. Here we want to apply this concept to the more general case (3).

Definition 1. Let a DAE of type (3) be given. Then we call this DAE a regular $D A E$ if there is a unique subbundle $(M, p, J)$ of $\mathbb{R} \times \mathbb{R}^{n}$ and a unique vector field $v: M \rightarrow \mathbb{R}^{n}$ on $M$ such that a differentiable mapping $c: I \rightarrow \mathbb{R}^{n}$ is a solution of the vector field $v$ if and only if $c$ is a solution of the given DAE. 
The manifold $M$ is then called the configuration space and the vector field $v$ the corresponding vector field of the DAE.

Example 2. Let us consider the DAE (2) where we assume that $D_{3} g(t, q,-i) \neq 0$ for all $(t, q, i) \in \mathbb{R}^{3}$. It is well known from state-space theory [6] that under this condition the DAE (2) has well defined solutions. We now show that the DAE is then regular too. For that reason we differentiate (2b) once and solve for $i^{\prime}$. Thus we obtain

$$
i^{\prime}=D_{3} g(t, q,-i)^{-1}\left[D_{1} g(t, q,-i)+D_{2} g(t, q,-i) i\right] \text {. }
$$

Now this equation and (2a) define a vector field on the manifold $M$ given by

$$
M=\left\{(t, q, i) \in \mathbb{R}^{n}: g(t, q,-i)=0\right\} .
$$

Obviously, in this case, the above-defined vector field is the corresponding vector field and the manifold $M$ is the configuration space of the DAE (2). However, in the following, we are interested in the case when $D_{3} g(t, q,-i)=$ 0 for some or all $(t, q, i) \in \mathbb{R}^{3}$. Indeed, it is this case for which our theory seems to be important.

Example 3. Let us now consider the DAE

$$
\begin{aligned}
x_{1}^{\prime}+x_{2}^{\prime} & =1, \\
x_{1} x_{2} & =0 .
\end{aligned}
$$

This DAE is not regular because the two solutions $c_{1}$ and $c_{2}$ defined by $c_{1}(t)=(t, 0)$ and $c_{2}(t)=(0, t)$ have the same initial value at $t=0$ but satisfy $D c_{1}(0) \neq D c_{2}(0)$. This is due to the fact that the set defined by $x_{1} x_{2}=0$ is not a manifold.

We now derive sufficient conditions for the regularity of a DAE. Furthermore, we state a technique by means of which we can obtain, for a given regular DAE, the configuration space $M$ and the corresponding vector field $v$. To start we associate with any DAE the corresponding set as defined below.

Definition 2. Let a DAE of type (3) be given. Then we call the set

$$
N=\left\{(t, x, p) \in J \times U \times \mathbb{R}^{n}: F(t, x, p)=0\right\}
$$

the corresponding set of the DAE.

Note, in Definition 2, the DAE (3) is considered as a nonlinear system of equations in the variables $t, x$, and $p$. For that reason the corresponding set $N$ of a DAE is a subset of $J \times U \times \mathbb{R}^{n}$. Clearly, a differentiable mapping $c: I \rightarrow \mathbb{R}^{n}$ is a solution of the DAE if and only if $(t, c(t), D c(t)) \in N$ for all $t \in I$. Therefore, with respect to the solutions of a DAE, we can con- 
sider the corresponding set instead. This fact is explored further in the next observation.

Observation 1. Let $N$ be the corresponding set of a given DAE (3). W'e consider the set $M_{1}$ defined by

$$
M_{1}=\operatorname{pr}_{1,2}(N),
$$

where $\operatorname{pr}_{1,2}: \mathbb{R} \times \mathbb{R}^{n} \times \mathbb{R}^{n} \rightarrow \mathbb{R} \times \mathbb{R}^{n}$ is the projection onto the first two components in $\mathbb{R} \times \mathbb{R}^{n} \times \mathbb{R}^{n}$.

The set $M_{1}$ is a subset of $\mathbb{R} \times \mathbb{R}^{n}$ and reflects the algebraic constraints on the solutions of the DA.E. Let us assume that the triple $\left(M_{1}, p, J\right)$ is a differentiable subbundle of $\mathbb{R} \times \mathbb{R}^{n}$. Clea:ly, under this assumption, a differentiable mapping $c: I \rightarrow \mathbb{R}^{n}$ is a solution of the given DAE if and only if

$$
(t, c(t), D c(t)) \in\left(N \cap S M_{1}\right)
$$

for all $t \in I$. $\left(N \cap S M_{1}\right)$ will be, in general, a subset of $N$. Therefore, we considered the set

$$
M_{2}=\operatorname{pr}_{1,2}\left(N \cap S M_{1}\right) .
$$

If the triple $\left(M_{2}, p, J\right)$ is now a differentiable subbundle of $\mathbb{R} \times \mathbb{R}^{n}$ as well, we conclude again that a differentiable mapping $c: I \rightarrow \mathbb{R}^{n}$ is a solution of the $\mathrm{DAE}$ if and only if

$$
(t, c(t), D c(t)) \in\left(N \cap S M_{2}\right)
$$

for all $t \in I$. This process can be continued as long as the triples $\left(M_{i}, p, J\right)$ with

$$
M_{i}=\operatorname{pr}_{1,2}\left(N \cap S M_{i-1}\right)
$$

are differentiable subbundles of $\mathbb{R} \times \mathbb{R}^{n}$ and may be stopped whenever $M_{i}=M_{i-1}$.

This observation leads us in a natural way to the following:

Definition 3. Let $N$ be the corresponding set of a given DAE (3). We define a family $\left(M_{i}\right)_{i=0, \ldots, s}$ of submanifolds $M_{i}$ of $\mathbb{R} \times \mathbb{R}^{n}$ by the recursion:

(i) $M_{0}=J \times U$,

(ii) $M_{i+1}=\operatorname{pr}_{1,2}\left(N \cap S M_{i}\right) \quad(i=0, \ldots, s-1)$,

where $s$ is the largest nonnegative integer such that the triples $\left(M_{i}, p, J\right)$ are differentiable subbundles and $M_{s-1} \neq M_{s}$. In case $M_{1}=J \times U$, we define $s=0$.

We call the family $\left(M_{i}\right)$ the family of constrained manifolds and the in "eger $s$ the degree of the given DAE. 
Remark 4. In [18] we have proved that $s \leq n$ for autonomous DAEs. Consequently, the degree $s$ of any nonautonomous DAE satisfies $s \leq n$ as well.

By means of the family of constrained manifolds we can state sufficient conditions for the regularity of a DAE.

Theorem 2. Let $N$ be the corresponding set, let $\left(M_{i}\right)$ be the family of constrained manifolds, and let s be the degree of a given DAE (3). Then this DAE is regular if the condition

C1: for all $(t, x) \in M_{s}$, there is a unique $p \in \mathbb{R}^{n}$ such that

$$
(t, x, p) \in\left(N \cap S M_{s}\right)
$$

is satisfied.

Under this condition, the configuration space $M$ of the $D A E$ is given by $M=M_{s}$, and the corresponding vector field $v: M \rightarrow \mathbb{R}^{n}$ is, for all $(t, x) \in M$, defined by

$$
(t, x, v(t, x)) \in\left(N \cap S M_{s}\right) .
$$

Furthermore, if additionally the condition

C2: $(N \cap S M)$ is a $C^{k}$-submanifold of $\mathbb{R} \times \mathbb{R}^{n} \times \mathbb{R}^{n}$ and, for all $(t, x, p) \in(N \cap S M), \operatorname{dim}(M)=\operatorname{dim}\left[\operatorname{pr}_{1,2}\left(T_{(t, x, p)}(N \cap S M)\right)\right]$

holds, then the corresponding vector field is of class $C^{k}$.

Proof. According to Observation 1, Condition $\mathrm{C} 1$ yields that a differentiable mapping $c: I \rightarrow \mathbb{R}^{n}$ is a solution of (3) if and only if, for all $t \in I$,

$$
(t, c(t), D c(t))=(t, c(t), v(t, c(t)),
$$

where $v: M \rightarrow \mathbb{R}^{n}$ is the vector field as defined in Theorem 2. Thus Condition $\mathrm{C} 1$ implies regularity of the DAE. Furthermore, Condition C2 and the implicit function theorem yield that the mapping $v$ is differentiable and of class $C^{k}$.

Once we have formulated sufficient conditions for the regularity of a DAE, it seems reasonable to state an existence and uniqueness theorem for regular DAEs. For example, such a theorem can be obtained just by "translating" Theorem 1 to regular DAEs.

Theorem 3. Let (3) be a regular DAE, let $M$ be the configuration space, and let $v$ be the corresponding vector field of this DAE. We assume the vector field $v$ to be of class $C^{k}, k>0$. Then there is a unique open set $D$ in $J \times M$ and a unique flow $H: D \rightarrow \mathbb{R}^{n}$ of class $C^{k}$ on $D$ such that a differentiable mapping $c: I \rightarrow \mathbb{R}^{n}$ with 
$c\left(t_{0}\right)=x_{0}$ is a solution of the DAE (3) if and only if

$$
c(t)=H\left(1 ; t_{0}, x_{0}\right)
$$

for all $t \in I$.

Naturally, the existence and uniqueress of solutions has been addressed in several other papers as well [4], [5], [16], [19]. For example, the following definition is due to Ca:npbell [4], [5]:

Definition 4. A DAE ( 3 ) is called solvable on a subset $\Gamma \times \Omega$ of $\mathbb{R} \times \mathbb{R}^{n}$ if there is a mapping $\mu: \Gamma \times \Omega_{i}^{0} \rightarrow \Omega$ on an open set $\Gamma \times \Omega^{0}$ of $\mathbb{R} \times \mathbb{R}^{k}, 0 \leq k \leq n$, such that

(i) if $c: I \rightarrow \mathbb{R}^{n}$ is a solution of the DAE whose graph lies in $\Gamma \times \Omega$, then $c(t)=\mu\left(t ; y_{0}\right)$ for all $t \in I$ and some $y_{0} \in \Omega^{0}$,

(ii) the graph of $\mu$ is an $(k+1)$-dimiensional submanifold of $\mathbb{R} \times \mathbb{R}^{n}$.

In case the corresponding vector ficld is differentiable, Theorem 3 y elds that regularity implies solvability of a I AE and that the integer $k$ is equal to the dimension of the configuration spase $M$. In fact, the notion of solvatility is equivalent in essence: to the notion of a flow on a manifold.

\section{A simple RC circuit}

In this section we investigate the DAE (2) which describes the time behavior of the simple RC circuit of Figure 1. Obviously, (2b) cannot be scilved globally for the variable $i$ in general. Consequently, the state equations [6] do not exist in the general case. Therefore, we now apply the theory of regular DAEs to the DAE (2).

By definition, the constrained manifold $M_{1}$ is given by

$$
M_{1}=\left\{(t, q, i) \in \mathbb{R} \times \mathbb{R}^{2}: 0=g(t, q,-i)\right\} .
$$

A point $(t, q, i) \in M_{1}$ is now an elemert of the set $M_{2}$ if and only if

$$
\left(N_{(t, q, i)} \cap S_{(t, q, i)} M_{1}\right) \neq \varnothing,
$$

where

$$
N_{(t, q, i)}=\left\{\left(q^{\prime}, i^{\prime}\right) \in \mathbb{R}^{2}: q^{\prime}=i\right\}
$$

and

$$
S_{(t, q, i)} M_{1}=\left\{\left(q^{\prime}, i^{\prime}\right): C=D_{1} g(t, q,-i\rangle+D_{2} g(t, q,-i) q^{\prime}-D_{3} g(t, q,-i) i^{\prime}\right\} .
$$

It is readily shown that we have

$$
\left(N_{(t, q, i)} \cap S_{(t, q, i)} M_{1}\right)=\varnothing
$$




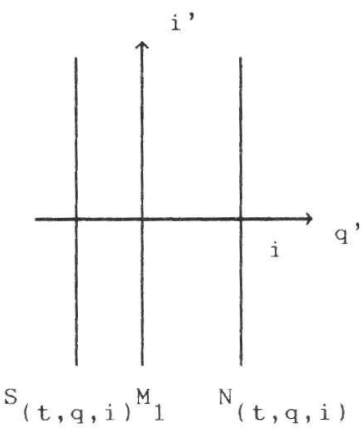

(a)

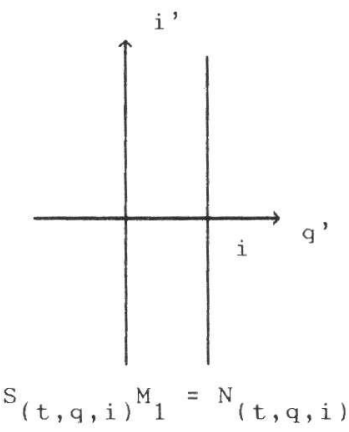

(b)

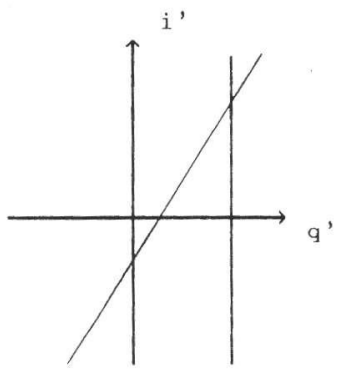

$\left.S_{(t, q, i}\right)_{1}^{M}{ }^{N}(t, q, i)$

(c)

Figure 2

if and only if $D_{3} g(t, q,-i)=0$ and $D_{2} g(t, q,-i)^{-1} D_{1} g(t, q,-i) \neq-i$. This is illustrated in Figure 2(a). In the following we call a point $(t, q, i) \in M_{1}$ that satisfies (5) a singular point.

Obviously, if (4) holds, then we have either

(i) $D_{3} g(t, q,-i)=0$ and $D_{2} g(t, q,-i)^{-1} D_{1} g(t, q,-i)=-i \quad$ (Figure 2(b)) or

(ii) $D_{3} g(t, q,-i) \neq 0$ (Figure $\left.2(\mathrm{c})\right)$.

For (ii) we obtain that the set

$$
N_{(t, q, i)} \cap S_{(t, q, i)} M_{1}
$$

contains exactly one element, while for (i) this set is diffeomorphic to the real line $\mathbb{R}$. Therefore, we call a point $(t, q, i) \in M_{1}$ regular if (ii) holds and degenerate if (i) holds.

According to Remark 4 in Section 3, we conclude that degree $s$ of the DAE (2) cannot exceed $s=2$.

We now discuss the results obtained so far by means of the specific voltage-current relations given in Figure 3(a)-(e). To simplify the discussion, we consider only time-invariant voltage-current relations. As a consequence, the constrained manifolds $M_{i}$ are trivial subbundles which we denote by

$$
M_{i}=\mathbb{R} \times Q_{i} .
$$

Clearly, by considering the manifolds $Q_{i}$ instead of the manifolds $M_{i}$, we can reduce the dimension of the problem by one. Therefore, to obtain good drawings, this fact is explored in the subsequent examples.

Example 4. First we consider the RC circuit of Figure 1 with the voltagecurrent relation of the nonlinear resistor as depicted in Figure 3(a). Clearly, 


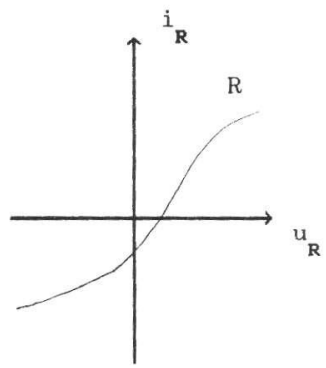

(a)

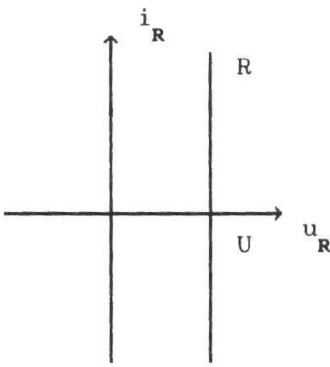

(d)

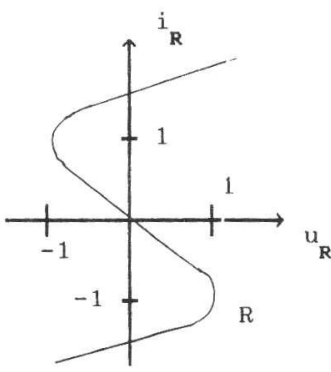

(b)

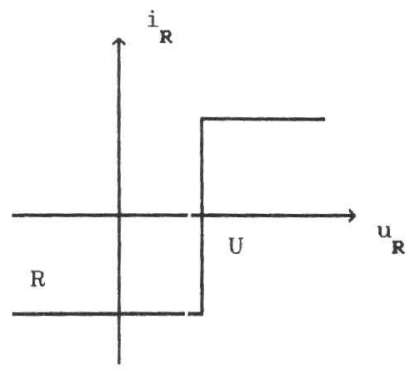

(e)

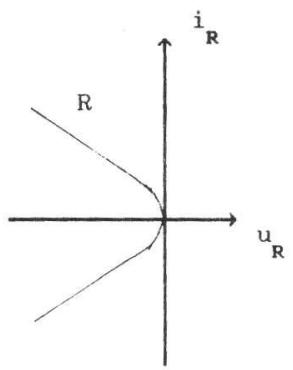

(c)

Figure 3

we have

$$
Q_{2}=Q_{1}^{\prime} ;
$$

that is, every point $(q, i) € Q_{1}$ is a regular point. Consequently, the DAE (2) is regular and of degree 1 .

Example 5. We now consider the voltage-current relation $R$ of Figure 3ib). We obtain

$$
\mathrm{Q}_{2}=Q_{1} \backslash\{(-1,-1),(1,1)\} ;
$$

- that is, $(-1,-1)$ and $(1,1)$ are singular points and all the other ponts belonging to $Q_{1}$ are regular. Thus the IJAE (2) is in this case regular and of degree 2. The vector field on $Q_{2}$ is depicted schematically in Figure 4.(a). Obviously, no solution can be extended beyond the two points $(-1,-1)$ and $(1,1)$. Therefore, such points are called impasse points [6]-[8]. Impasse points are an important phenomenon found in many circuits (see, for example, [7]) in connestion with the so-called jump behavior.

Example 6. Let the voltage-current relation $R$ be given by Figure 3(c) 'with $g\left(u_{R}, i_{R}\right)=i_{R}^{2}+u_{R}$. Sinilar to Examplı 4 we obtain

$$
Q_{2}=Q_{1} \text {. }
$$




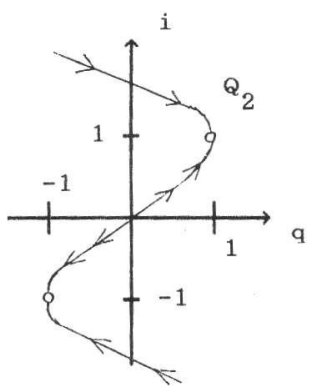

(a)

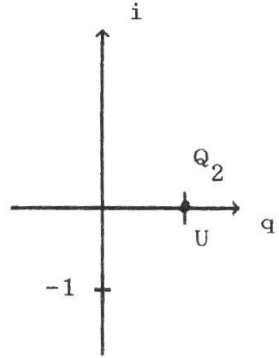

(b)

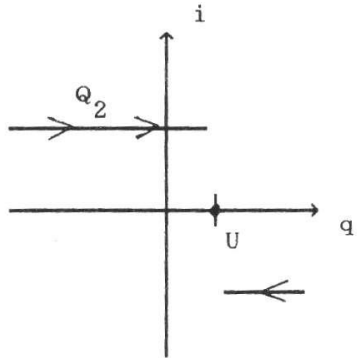

(c)

Figure 4

However, the point $(0,0)$ is a degenerate point. Hence Condition $\mathrm{C} 1$ of Theorem 2 does not hold because

$$
N_{(0,0)} \cap T_{(0,0)} Q_{2}
$$

is diffeomorphic to the real line $\mathbb{R}$. The two mappings $c_{i}: I \rightarrow \mathbb{R}^{2}(i=1,2)$ defined by

$$
c_{1}(t)=(0,0) \quad \text { and } c_{2}(t)=\left(-t^{2} / 4,-t / 2\right) \quad(t \in I)
$$

are solutions of the DAE (2) through the point $(0,0)$. Now because

$$
D c_{1}(0) \neq D c_{2}(0),
$$

we conclude that the DAE (2) with the characteristic of Figure 3(c) cannot be regular.

Example 7. We now consider the voltage-current relation $R$ of Figure 3(d). The resulting circuit is equivalent to the circuit which we obtain by replacing the nonlinear resistor by a dc voltage source $u(t)=U$. With the exception of the point $(U, 0)$ all the other points belonging to $Q_{1}$ are singular and we therefore get

$$
Q_{2}=\{(U, 0)\} .
$$

The point $(U, 0)$ is degenerate. But because $\operatorname{dim}\left(Q_{2}\right)=0$, we have

$$
\left(N_{(U, 0)} \cap T_{(U, 0)} Q_{2}\right)=\{(0,0)\} .
$$

Accordingly, Condition C1 of Theorem 2 is satisfied and the DAE (2) is regular and of degree 2 (Figure 4(b)).

Example 8. Finally, consider the voltage-current relation $R$ given in Figure 3(e). The circuit specified by this characteristic is closely related to systems 
with variable structure and. sliding mode [3]. So far such systems have beer modeled by differential inclusions [3]. By means of this example we argut: that those systems can be considered as higher-index DAEs as well. It should be noted that, in contrast to [3], we have to assume differentiability of the characteristic $R$. However, this is not a severe restriction and can be avoided if, instead of tangent spaces, the more general notion of one-sided tangent spaces [2] is used.

The constrained manifold $Q_{2}$ is depicted in Figure 4(c). Again, the point $(U, 0)$ is a degenerate point. However, the same reasoning as in Example 7 yields that the DAE (2) is regular and of degree 2. We like to remark that the sliding mode is in this case the solution $c: I \rightarrow \mathbb{R}^{2}$ with $c(t)=(U, 0)$ for all $t \in I$.

\section{DAEs of index $m$}

In several papers [4], [5], [9], [14], [16] the properties of DAEs are characterized by an integer called the index. In this section we compare the notion of index with our concepts of regularity and degree. The fundamental idea, behind the index definition in [4], [5], [9], [14], and [17], is to differentiate the mapping $F$ of (3) several times and to investigate the resulting overdetermined system of equations. Specifically:

Let a DAE of type (3) be given. Then, for any nonnegative integer $m$, ve consider, as long as the mappings involved are defined and differentiable, the nonlinear system of equations

$$
\begin{gathered}
F_{0}(t, x, p)=0, \\
F_{1}(t, x, p)=0, \\
\vdots \\
F_{m}\left(t, x, p_{i}=0\right.
\end{gathered}
$$

with

$$
\begin{aligned}
F_{0}(t, x, p) & :=F(t, x, p), \\
F_{i+1}(t, x, p) & :==Q_{i}(t, x, p)\left[D_{1} F_{i}(t, x, p)+D_{2} F_{i}(t, x, p) p\right],
\end{aligned}
$$

where $Q_{i}(t, x, p)$ is a projection along $\operatorname{im}\left[D_{3} F_{i}(t, x, p)\right]$. Let us write the system of equations (6) in the form

$$
G_{m}(t, x, p)=0
$$

with $t \in J, x \in U, p \in \mathbb{R}^{n}, G_{m}: J \times U \times \mathbb{R}^{n} \rightarrow\left(\mathbb{R}^{n}\right)^{m+1}$. We call the mapping $G_{m}$ the derivative array of corder $m$.

As long as the derivative array $G_{\mathrm{k}}$ of order $k$ is defined, we associate wi $h$ it the two sets

$$
L_{k}=\left\{(t, x, p) \in J \times U \times \mathbb{R}^{n}: G_{k}(t, x, p)=0\right\}
$$


and

$$
P_{k}=\operatorname{pr}_{1,2}\left(L_{k}\right)
$$

Using a terminology which is appropriated for our purposes, we state the index definition, as given in [4], [5], [9], [14], and [16], in the following way:

Definition 5. Let a DAE of type (3) be given. The index of the DAE, in the sense of [4], [5], [9], [14], and [16], is the smallest nonnegative integer $m$ such that

(i) the triple $\left(P_{m}, p, J\right)$ is a $C^{1}$-subbundle of $J \times U$,

(ii) for any $(t, x) \in P_{m}$ there exists a unique $p \in \mathbb{R}^{n}$ with $(t, x, p) \in L_{m}$.

Of course, such an integer does not exist in any case. But if the index can be defined for a given DAE (3), then (ii) defines a unique mapping $g: P_{m} \rightarrow \mathbb{R}^{n}$. Furthermore, by definition of the mapping $g$, a differentiable mapping $c: I \rightarrow \mathbb{R}^{n}$ is a solution of (3) if an only if $c$ is a solution of the ODE

$$
x^{\prime}=g(t, x) \quad\left((t, x) \in P_{m}\right)
$$

defined on the manifold $P_{m}$. Thus we obtain the following:

Proposition 1. Let (3) be a DAE of index $m$. Then this DAE is regular. Furthermore, the configuration space $M$ is given by $M=P_{m}$ and the corresponding vector field $v: M \rightarrow \mathbb{R}^{n}$ is given by

$$
(t, x, v(t, x)) \in L_{m}
$$

for all $(t, x) \in M$.

In the remainder of this section we compare the notions of index and degree and show that the constrained manifolds $M_{i}$ are identical with the sets $P_{i}$. Thus the derivative arrays allow for a computation of the constrained manifolds.

Observation 2. Let (3) be a DAE of degree $s$, let $N$ be the corresponding set, and let $\left(M_{i}\right)$ be the family of constrained manifolds. Furthermore, we assume that the derivative arrays exist up to and including order $s$. Obviously, by definition of the derivative array $G_{0}$, we have

$$
L_{0}=N
$$

and thus

$$
P_{0}=M_{1} .
$$

Now the set $N \cap S M_{1}$ can be equivalently written as

$$
N \cap S M_{1}=\left\{(t, x, p): \exists q \in \mathbb{R}^{n} \text { such that }(t, x, p, p, q) \in S N\right\} .
$$


Because the set $S N$ is given by the solutions of the following system of equations,

$$
\begin{aligned}
& 0=F(t, x, p), \\
& 0=D_{1} F(t, x, p)+D_{2} F(t, x, p) x^{\prime}+D_{3} F(t, x, p) p^{\prime},
\end{aligned}
$$

we obtain that $N \cap S M_{1}$ is given by the solutions of the following system of equations:

$$
\begin{aligned}
& 0=F(t, x, p), \\
& 0=Q(t, x, p)\left[D_{1} F(t, x, p)+D_{2} F(t, x, p) p\right],
\end{aligned}
$$

where $Q(t, x, p)$ is a projection along $\operatorname{im}\left[D_{3} F(t, x, p)\right]$. However (7) is equivalent to the derivative array of order 1 . Thus we have

$$
L_{1}=N \cap S M_{1}
$$

and

$$
P_{1}=M_{2}
$$

This process can be continued and we obtain the following:

Proposition 2. Let (3) be a DAE of degree s. Furthermore, let the derivative arrays exist up to and including order s. Then we have

$$
L_{i}=N \cap S M_{i}
$$

and

$$
P_{i}=M_{i+1}
$$

for all $i \in\{0, \ldots, s\}$.

Finally, we state rark-conditions on the derivative arrays that ensure the regularity of a DAE. (Similar conditions are given in [4] and [5].)

Theorem 4. Let a DAE of type (3) where the mapping $F$ is of class $C^{r}, r>0$, be given. Let us assume that there is a nonnegative integer $k$ such that the derivative arrays $G_{m}$ of order $m, m \leq k$, exist and satisfy the conditions:

(i) For $m<k,\left[\begin{array}{lll}D_{2} & G_{m} & D_{3} G_{m}\end{array}\right](t, x, p)$ and $D_{3} G_{m}(t, x, p)$ have constant rank for all $(t, x, p) \in J \times U \times \mathbb{R}^{n}$.

(ii) The row echelon form of $D_{3} G_{k}(t, x, p)$ is

$$
\left[\begin{array}{c}
I_{n} \\
0
\end{array}\right]
$$

independent of $(t, x, p) \in J \times U \times \mathbb{R}^{n}$. 
Then this DAE is regular and of degree $s$ where $s$ is the smallest nonnegative integer such that (i) and (ii) hold.

Proof. For $m<k$, Condition (i) implies that for all $\left(t_{0}, x_{0}, p_{0}\right) \in L_{m}$ there is a neighborhood $V_{m}$ of $\left(t_{0}, x_{0}, p_{0}\right)$ in $J \times U \times \mathbb{R}^{n}$ such that, with $W_{m}=p_{1,2}\left(V_{m}\right)$,

$$
L_{m} \cap V_{m}=\left\{(t, x, p) \in V_{m}: 0=h_{m}(t, x, p), 0=g_{m}(t, x)\right\}
$$

and

$$
P_{m} \cap W_{m}=\left\{(t, x) \in W_{m}: 0=g_{m}(t, x)\right\},
$$

where $\quad h_{m}: V \rightarrow \mathbb{R}^{p_{m}}$ and $g_{m}: W \rightarrow \mathbb{R}^{q_{m}}$ are $C^{r-m}$-mappings with a $\operatorname{rank}\left[D_{2} g_{m}(t, x)\right]=q_{m}$ and $\operatorname{rank}\left[D_{3} h_{m}(t, x, p)\right]=p_{m}$ for all $(t, x, p) \in V_{m}$. Thus the sets $P_{m}$ are differentiable subbundles. Condition (ii) implies that there is a mapping $h: W_{k-1} \rightarrow \mathbb{R}^{n}$ of class $C^{r-k}$ such that

$$
L_{k} \cap V_{k}=\left\{(t, x, p\} \in V_{k}: p=h_{k}(t, x), 0=g_{k-1}(t, x)\right\},
$$

where $V_{k}=W_{k-1} \times \mathbb{R}^{n}$. Thus the DAE (3) is regular and of index $s$.

\section{Concluding remarks}

By means of a simple RC circuit we have shown that the properties of DAEs can be quite complex. Therefore, in this and other papers [17], [18] we have stated a method for a characterization of DAEs in differential-geometric terms. However, many open questions still remain in connection with this method. For example, further studies about the relation of DAEs to vector fields on special fields, like bifurcation and stability theory (see, e.g., [8]), are still needed, and necessary conditions for the differentiability of the constrained manifolds should be derived in terms of transversality [11].

\section{Acknowledgments}

We are indebted to S. L. Campbell and A. Reibiger who read significant parts of the paper, identified errors, and provided helpful and insightful feedback.

\section{References}

[1] Abraham, R.; Marsden, J. E.; Ratiu, M.: Manifolds, Tensor Analysis, and Applications. Reading, Mass.: Addison-Wesley, 1983.

[2] Alekseev, V. M.; Tikhomirov, V. M.; Fomin, S. V.: Optimal Control. New York: Consultants Bureau, 1987.

[3] Aubin, J. P.; Cellina, A.: Differential Inclusions. Berlin: Springer-Verlag, 1981.

[4] Brenan, K. E.; Campbell, S. L.; Petzold, L. R.: The Numerical Solution of Initial Value Problems in Differential-Algebraic Equations. Amsterdam: North-Holland, 1989. 
[5] Campbell, S. L.: A Computa ional Method for Cieneral Higher Index Nonlinear Singular Systems of Differential Equations. CRSC Technical Report 063087-01, North Carolina State University.

[6] Chua, L. O.: Introduction to Nonlinear Network Theory. New York: McGraw-Hill, 1969.

[7] Chua, L. O.; Deng, A.: Impasse points. Interrat. J. Circuit Theory Appl., 17 (1989), 213-235.

[8] Chua, L. O.; Oka, H.: Normal forms for constrained nonlinear differential equations. Part II: bifurcation. IEEE Trans. Circuits and Systemis, 36 (1989), 71-88.

[9] Gear, C. W.: DAEs index transformations. SIAM J. Sci. Statist. Comput., 9 (1988), 39-47.

[10] Griepentrog, E.; März, R.: L'AEs and Their Numerical Treatment. Leipzig: Teubner, 1986.

[11] Haggman, B. C.; Bryant, P R.: Solutions of singular constrained differential equations. IEEE Trans. Circuits and Sj'stems, 31 (1984), 1015-1029.

; [12] Hairer, E.; Lubich, Ch.; Roche, M.: The Numerical Solution of Differential-Algebraic: Equations by Runge-Kutta Methods. Lecture Notes in Mathematics, vol. 1409. Berlin: Springer-Verlag, 1989.

[13] Lang, S.: Introduction to Differentiable Manifolds. New York: Wiley, 1962.

- [14] Lötstedt, P.; Petzold, L. R.: Numerical solution of nonlinear differential equations with algebraic constraints I. Ma.h. Comp., 46 (1986), 491-516.

[15] Potra, F. A.; Rheinboldt, W'. C.: Differential-Geometric Techniques for Solving Differential-Algebraic Equations. Tichnical Report ICMA-89-143, University of Pittsburgh, 1985.

[16] Rabier, P. J.; Rheinboldt, W' C.: A General Exisitence and Uniqueness Theorem for DAE:3. Technical Report ICMA-9()-145, University of Pittsburgh, 1990.

[17] Reich, S.: Beitrag zur Theorie der Algebrodifferentialgleichungen. Ph.D. Thesis, Technical University Dresden, 1990.

[18] Reich, S.: On a geometr.c interpretation of differential-algebraic equations. Circuits Systems Signal Process., 9 (1990), 367-382.

[19] Rheinboldt, W. C.: Differential-algebraic systems as differential equations on manifolds. Math. Comp., 43 (1984), 14.73-1482. 